www.mdpi.com/journal/marinedrugs

\title{
Communication
}

\section{Spirobisnaphthalenes from the Mangrove-Derived Fungus Rhytidhysteron sp. AS21B}

\author{
Khanitha Pudhom ${ }^{1, *}$, Thapong Teerawatananond ${ }^{1}$ and Supichar Chookpaiboon ${ }^{2}$ \\ 1 Department of Chemistry, Faculty of Science, Chulalongkorn University, Bangkok 10330, \\ Thailand; E-Mail: thapong_sthc@hotmail.com \\ 2 Program in Biotechnology, Faculty of Science, Chulalongkorn Universtiy, Bangkok 10330, \\ Thailand; E-Mail: bubu_keaw@hotmail.com \\ * Author to whom correspondence should be addressed; E-Mail: khanitha.p@chula.ac.th; \\ Tel.: +66-2218-7641; Fax: +66-2254-1309.
}

Received: 6 January 2014; in revised form: 8 February 2014 / Accepted: 18 February 2014 / Published: 6 March 2014

\begin{abstract}
Three new spirobisnaphthalenes (1-3) were isolated from the mangrove-derived fungus Rhytidhysteron sp., together with five known derivatives (4-8). The structures of the compounds were established on the basis of extensive spectroscopic data, and the relative configurations of their stereogenic carbons were determined by a single-crystal X-ray crystallographic analysis. Compounds 3-5 displayed cytotoxicity against both cancer cell lines, MCF-7 and CaSki, while 2 was active only on CaSKi cells.
\end{abstract}

Keywords: endophytic fungus; Rhytidhysteron sp.; spirobisnaphthalene; cytotoxicity

\section{Introduction}

Spirobisnaphthalenes, a series of compounds consisting of two naphthalene-derived $\mathrm{C}_{10}$ units bridged through a spiroketal linkage, have been mainly isolated from fungi [1-5]. This class of compounds is of great interest as potential leads for medicinal chemistry, since they have interesting structures and a variety of biological activities such as antibacterial, antifungal, anticancer, and antileishmanial activities [4-7].

Endophytic fungi are known as a prolific source for the discovery of structurally interesting and biologically active metabolites [8-11]. Among plant-derived fungi, those associated with the trees growing up in mangrove areas have received much attention from medicinal chemists owing to the 
unique ecosystem [12]. In our continued investigation into new bioactive compounds from Thai mangrove-derived fungi, we describe the isolation and structure elucidation of three new spirobisnaphthalenes, rhytidones A-C (1-3), together with five known derivatives from an endophytic Rhytidhysteron sp. fungus. In addition, all isolated compounds were evaluated for their cytotoxic activities against human cancer cell lines.

\section{Results and Discussion}

The Rhytidhysteron sp. fungus was cultured in malt extract broth (MEB) under static conditions for 21 days. The EtOAc crude extract of the culture broth was successively subjected to Sephadex LH-20 and silica gel column chromatography to afford three new spironaphthalenes, rhytidones A-C (1-3), and five known analogues including MK3018 (4), palmarumycin CR1 (5), CJ-12,372 (6), 4-O-methyl-CJ-12,372 (7) and 4-O-methyl-CJ-12,371 (8) as shown in Figure 1. The structures of the known compounds were determined by comparison of their NMR spectroscopic data with those in the literature [13-16].

Figure 1. Structures of compounds 1-8 isolated from fungus Rhytidhysteron sp.
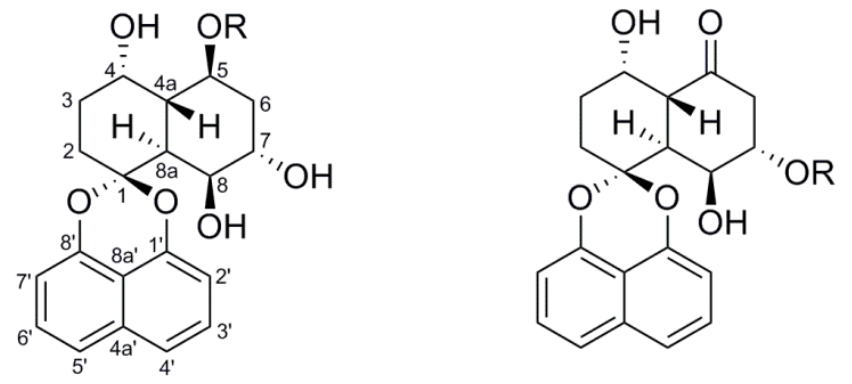

$$
\begin{array}{ll}
1 & R=H \\
1 a & R=A c
\end{array}
$$

$$
\begin{array}{ll}
2 & \mathrm{R}=\mathrm{H} \\
3 & \mathrm{R}=\mathrm{Me}
\end{array}
$$<smiles>[R]C1=CC=C(O)C2C1C(O)CCC21Oc2cccc3cccc(c23)O1</smiles>

$$
\begin{array}{ll}
4 & \mathrm{R}=\mathrm{O} \\
\mathbf{5} & \mathrm{R}=\mathrm{H}, \mathrm{OH}
\end{array}
$$<smiles>[R2]c1ccc(O)c2c1C1(CCC2[R2])Oc2cccc3cccc(c23)O1</smiles>

$$
\begin{array}{ll}
6 & \mathrm{R}_{1}=\mathrm{H}, \mathrm{R}_{2}=\mathrm{OH} \\
7 & \mathrm{R}_{1}=\mathrm{Me}, \mathrm{R}_{2}=\mathrm{OH} \\
8 & \mathrm{R}_{1}=\mathrm{Me}, \mathrm{R}_{2}=\mathrm{H}
\end{array}
$$

Rhytidone A (1) was obtained as a light brown powder and its molecular formula was established as $\mathrm{C}_{20} \mathrm{H}_{22} \mathrm{O}_{6}$ from HRESIMS at $m / z 381.1319[\mathrm{M}+\mathrm{Na}]^{+}$(calcd 381.1314), implying 10 degrees of unsaturation. Detailed analysis of the ${ }^{1} \mathrm{H},{ }^{13} \mathrm{C}$ and HSQC NMR data revealed the presence of six methine carbons (four oxygenated), three methylene carbons, one doubly oxygenated quaternary carbon $\left(\delta_{\mathrm{C}} 104.4\right)$ and 10 aromatic carbons. In the ${ }^{1} \mathrm{H}-{ }^{1} \mathrm{H}$ COSY spectrum, homonuclear coupling 
correlations of $\mathrm{H}-2^{\prime} / \mathrm{H}-3^{\prime}$ and $\mathrm{H}-3^{\prime} / \mathrm{H}-4^{\prime}$, as well as correlations of $\mathrm{H}-5^{\prime} / \mathrm{H}-6^{\prime}$ and $\mathrm{H}-6^{\prime} / \mathrm{H}-7^{\prime}$ indicated the presence of two isolated three-proton spin systems corresponding to the C-2'-C-4' and C-5'-C-7' subunits of 1 , which displayed the ortho coupling constant with $J$ values of 7.2, 7.6 and 8.0 Hz. The HMBC correlations of $\mathrm{H}-2^{\prime} / \mathrm{C}-1^{\prime}, \mathrm{H}-2^{\prime} / \mathrm{C}-8 \mathrm{a}^{\prime}, \mathrm{H}-3^{\prime} / \mathrm{C}-4 \mathrm{a}^{\prime}, \mathrm{H}-6^{\prime} / \mathrm{C}-4 \mathrm{a}^{\prime}, \mathrm{H}-7^{\prime} / \mathrm{C}-8^{\prime}$ and $\mathrm{H}-7^{\prime} / \mathrm{C}-8 \mathrm{a}^{\prime}$ led to the attachment of both subunits at $\mathrm{C}-4 \mathrm{a}^{\prime}$ and $\mathrm{C}-8 \mathrm{a}^{\prime}$, suggesting the presence of a naphthalene moiety. In addition, the chemical shifts of the nonprotonated carbons $\mathrm{C}-1^{\prime}$ and $\mathrm{C}-8^{\prime}$ at $\delta_{\mathrm{C}} 147.4$ and 145.9 , respectively, were indicative of a 1,8-dioxynaphthalene moiety. Based on the above evidence, compound 1 was recognized as a member of the spirobisnaphthalene, characteristic of a 1,8-dioxynaphthalene linked with the second half of the molecule via a spiroketal carbon. The remaining part of the molecule was mainly elucidated by analysis of ${ }^{1} \mathrm{H}-{ }^{1} \mathrm{H}$ COSY and $\mathrm{HMBC}$ data. The COSY correlations confirmed the presence of the new extended spin system corresponding to the C-2-C-8 subunit (including C-4a and C-8a) (Figure 2a). HMBC correlations of H-2 and H-8a with C-1 led to the attachment of the spiroketal bridge carbon to the last subunit at C-2 and C-8a. Moreover, four exchangeable protons, observed at $\delta_{\mathrm{H}} 4.41,4.81,3.85,4.22$, were assigned to OH-4, OH-5, OH-7 and $\mathrm{OH}-8$, respectively, by their COSY correlations with their vicinal protons (Figure 2a). The relative configuration of 1 was determined through single-crystal X-ray diffraction analysis of its 5 -acetate derivative (1a), obtained from acetylation of $\mathbf{1}$ with acetic anhydride in the presence of a catalytic amount of DMAP. The perspective ORTEP plot of $\mathbf{1 a}$ is shown in Figure $2 \mathrm{~b}$.

Figure 2. (a) Key HMBC and ${ }^{1} \mathrm{H}-{ }^{1} \mathrm{H}$ COSY correlations of rhytidone A (1); (b) ORTEP diagram of the 5 -acetate derivative of $\mathbf{1}(\mathbf{1 a})$.

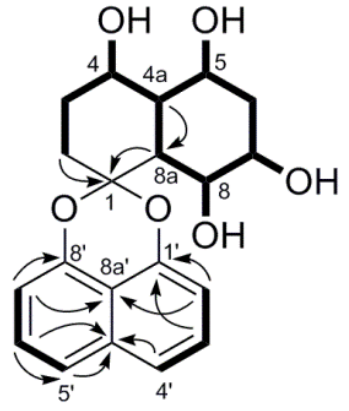

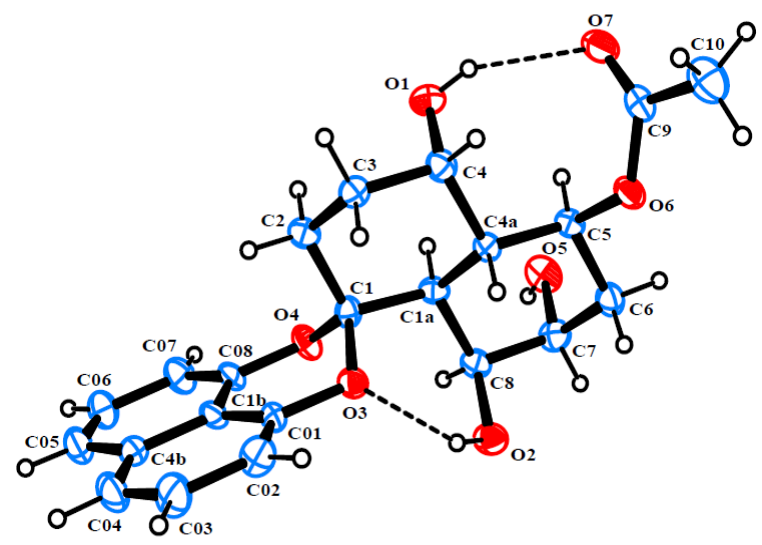

b

Rhytidone B (2) was isolated as colorless crystals. Its HRESIMS spectrum exhibited a pseudo-molecular ion at $\mathrm{m} / \mathrm{z} 379.1153\left([\mathrm{M}+\mathrm{Na}]^{+}\right.$, calcd 379.1158), consistent with the molecular formula $\mathrm{C}_{20} \mathrm{H}_{20} \mathrm{O}_{6}$. The NMR data of 2 also displayed characteristic signals associated with a spirobisnaphthalene, including a 1,8-dioxynaphthalene moiety and a spiroketal bridge carbon. Moreover, its NMR data (Table 1) were similar to those of $\mathbf{1}$, except for the replacement of one oxygenated methine carbon in 1 by a new ketone carbon $\left(\delta_{\mathrm{C}} 212.2\right)$. The $-\mathrm{CH}_{2}(2)-\mathrm{CH}_{2}(3)-\mathrm{CH}(4)$ $\mathrm{OH}-\mathrm{CH}(4 \mathrm{a})-\mathrm{CH}(8 \mathrm{a})-\mathrm{CH}(8) \mathrm{OH}-\mathrm{CH}(7)-\mathrm{OH}-\mathrm{CH}_{2}(6)-$ subunit was established by analysis of ${ }^{1} \mathrm{H}-{ }^{1} \mathrm{H}$ COSY. HMBC correlations from $\mathrm{H}-4 \mathrm{a}$ and $\mathrm{H}_{2}-6$ to the ketone carbon clearly indicated the location of 
the ketone carbonyl at C-5. Ultimately, the structure and relative configuration of 2 was clarified by single-crystal X-ray diffraction analysis (Figure 3).

Table 1. NMR spectroscopic data of compounds $\mathbf{1}-\mathbf{3}$.

\begin{tabular}{|c|c|c|c|c|c|c|}
\hline \multirow{2}{*}{ No. } & \multicolumn{2}{|l|}{$1^{a}$} & \multicolumn{2}{|l|}{$2^{b}$} & \multicolumn{2}{|l|}{$3^{b}$} \\
\hline & $\delta_{\mathrm{H}}(J$ in $\mathrm{Hz})$ & $\delta_{\mathrm{C}}$ & $\delta_{\mathrm{H}}(J$ in $\mathrm{Hz})$ & $\boldsymbol{\delta}_{\mathrm{C}}$ & $\delta_{\mathrm{H}}(J$ in $\mathrm{Hz})$ & $\boldsymbol{\delta}_{\mathrm{C}}$ \\
\hline 1 & - & 104.4 & - & 104.4 & - & 104.4 \\
\hline 2 & $1.73, \mathrm{~m}$ & 25.6 & $1.95, \mathrm{~m}$ & 25.6 & $1.94, \mathrm{~m}$ & 25.6 \\
\hline 3 & $1.53, \mathrm{~m}$ & 28.2 & $\begin{array}{l}1.75, \mathrm{~m} \\
1.66, \mathrm{~m}\end{array}$ & 26.5 & $\begin{array}{l}1.72, \mathrm{~m} \\
1.62, \mathrm{~m}\end{array}$ & 26.6 \\
\hline 4 & 4.16 , br s & 61.2 & 4.54 , br s & 62.9 & 4.52 , br s & 62.9 \\
\hline $4 a$ & 1.97, ddd $(12.8,10.0,2.4)$ & 42.9 & $3.31, \mathrm{~d}(13.6)$ & 49.0 & 3.27 , dd $(13.2,1.6)$ & 48.9 \\
\hline 5 & 3.70 , br s & 68.8 & - & 212.2 & - & 211.8 \\
\hline 6 & $1.75, \mathrm{~m}$ & 35.7 & $\begin{array}{c}3.13, \text { br s } \\
2.47, \text { dd }(14.4,2.8)\end{array}$ & 44.2 & $\begin{array}{c}3.05, \mathrm{~m} \\
2.60, \mathrm{dd}(14.4,2.0)\end{array}$ & 41.2 \\
\hline 7 & 4.21 , br s & 66.9 & $4.43, \mathrm{t}(3.2)$ & 71.3 & $3.89, \mathrm{~m}$ & 80.1 \\
\hline 8 & $3.93, \mathrm{~m}$ & 63.3 & $4.77, \mathrm{~d}(3.2)$ & 67.4 & 4.88 , br d (3.6) & 65.1 \\
\hline $8 \mathrm{a}$ & $2.44, \mathrm{dd}(12.8,1.6)$ & 38.6 & 3.17 , br s & 41.5 & $3.03, \mathrm{~m}$ & 41.9 \\
\hline $1^{\prime}$ & - & 146.5 & - & 147.3 & - & - \\
\hline $2^{\prime}$ & $6.96, \mathrm{~d}(7.2)$ & 109.4 & $6.94, \mathrm{~d}(7.2)$ & 109.8 & $6.96, \mathrm{~d}(7.6)$ & 109.8 \\
\hline $3^{\prime}$ & $7.45, \mathrm{t}(7.6)$ & 127.6 & $7.43, \mathrm{t}(8.0)$ & 127.8 & $7.44, \mathrm{t}(7.6)$ & 127.7 \\
\hline $4^{\prime}$ & $7.50, \mathrm{~d}(8.0)$ & 119.7 & $7.53, \mathrm{~d}(8.4)$ & 121.5 & $7.48, \mathrm{~d}(8.4)$ & 120.5 \\
\hline $4 a^{\prime}$ & - & 133.6 & - & 134.2 & - & 134.2 \\
\hline $5^{\prime}$ & $7.52, \mathrm{~d}(8.0)$ & 120.1 & $7.49, \mathrm{~d}(8.4)$ & 120.5 & $7.53, \mathrm{~d}(8.4)$ & 121.4 \\
\hline $6^{\prime}$ & $7.45, \mathrm{t}(7.6)$ & 127.5 & $7.43, \mathrm{t}(8.0)$ & 127.1 & $7.42, \mathrm{t}(7.6)$ & 127.1 \\
\hline $7^{\prime}$ & $6.94, \mathrm{~d}(7.2)$ & 108.8 & $6.95, \mathrm{~d}(7.2)$ & 109.6 & $6.93, \mathrm{~d}(7.6)$ & 109.6 \\
\hline $8^{\prime}$ & - & 147.6 & - & 145.9 & - & 145.9 \\
\hline $8 a^{\prime}$ & - & 113.3 & - & 113.8 & - & 113.9 \\
\hline $4-\mathrm{OH}$ & 3.70 , br s & - & - & - & - & - \\
\hline $5-\mathrm{OH}$ & $4.81, \mathrm{~d}(2.8)$ & - & - & - & - & - \\
\hline $7-\mathrm{OH}$ & $3.85, \mathrm{~d}(2.4)$ & - & - & - & - & - \\
\hline $8-\mathrm{OH}$ & 4.21 , br s & - & $3.77, \mathrm{~s}$ & - & $3.71, \mathrm{~s}$ & - \\
\hline 7-OMe & - & - & - & - & $3.43, \mathrm{~s}$ & 56.9 \\
\hline
\end{tabular}

${ }^{\mathrm{a}}$ Measured in DMSO- $\mathrm{d}_{6} ;{ }^{\mathrm{b}}$ measured in $\mathrm{CDCl}_{3}$.

Rhytidone C (3), obtained as colorless crystals, gave the molecular formula $\mathrm{C}_{21} \mathrm{H}_{22} \mathrm{O}_{6}$, as established by HRESIMS ( $\mathrm{m} / \mathrm{z} 393.1315\left([\mathrm{M}+\mathrm{Na}]^{+}\right.$, calcd. 393.1314). The NMR data of 3 (Table 1$)$ were very similar to those of 2 , except for the presence of an additional methoxy group $\left(\delta_{\mathrm{H}} 3.43 \mathrm{~s}\right.$, $\left.\delta_{\mathrm{C}} 56.9\right)$. Strong $\mathrm{HMBC}$ correlation from methoxyl protons to $\mathrm{C}-7$ at $\delta_{\mathrm{C}} 80.1$ indicated the attachment of the methoxyl group at C-7. Compound $\mathbf{3}$ was found to have the same configuration as in $\mathbf{2}$, which was also determined by single-crystal X-ray diffraction analysis (Figure 4). 
Figure 3. ORTEP diagram of rhytidone B (2).

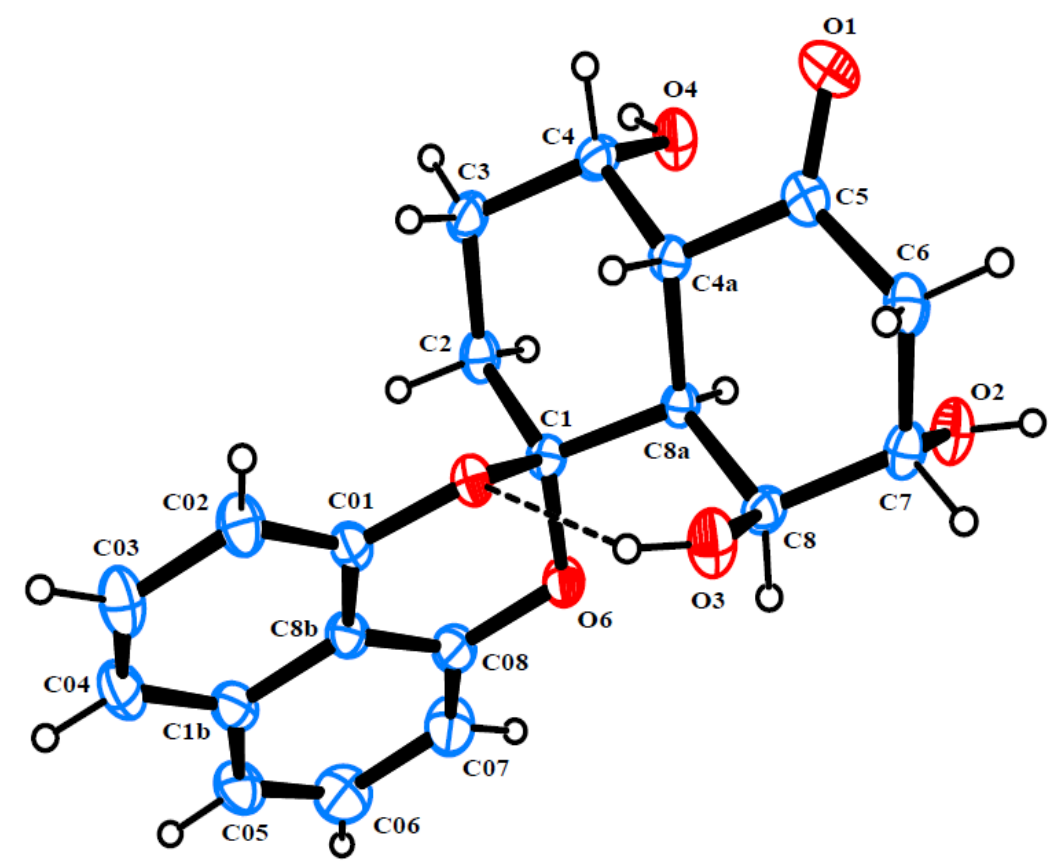

Figure 4. ORTEP diagram of rhytidone $\mathrm{C}(3)$.

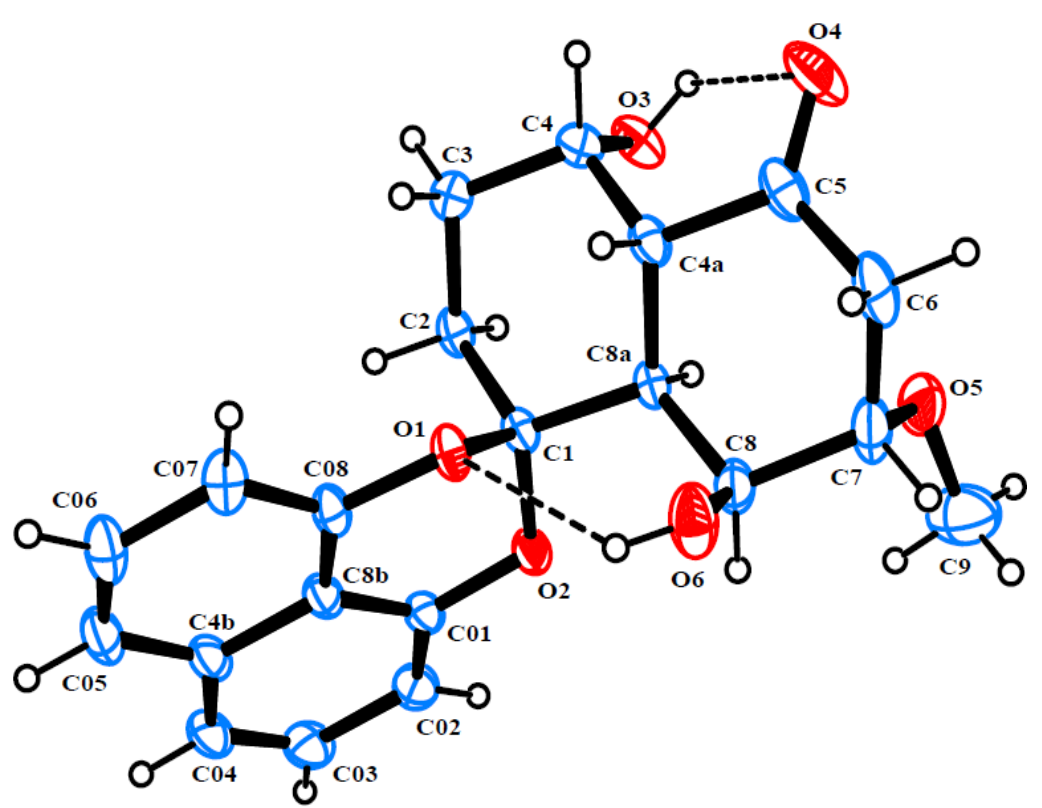

Cytotoxic activity of isolated compounds against human breast cancer (MCF-7) and cervical carcinoma (CaSki) cell lines was evaluated by the MTT method (Table 2) [17]. Compounds 3-5 showed moderate activity against both cell lines with $\mathrm{IC}_{50}$ values between 14.47 and $25.59 \mu \mathrm{M}$, while compound 2 was active only for CaSki with $\mathrm{IC}_{50}$ value of $22.81 \mu \mathrm{M}$. It should be noted that compounds 6-8, possessing an additional aromatic ring in the structure, did not exhibit any significant activity against the cell lines tested $\left(\mathrm{IC}_{50}>10 \mu \mathrm{g} / \mathrm{mL}\right)$.

More details are available at the Supplementary Information. 
Table 2. Cytotoxicity of compounds $\mathbf{2}-\mathbf{5}$ against human breast and cervical cancer cell lines.

\begin{tabular}{ccc}
\hline \multirow{2}{*}{ Compound } & \multicolumn{2}{c}{$\mathbf{I C}_{\mathbf{5 0}}(\boldsymbol{\mu M})$} \\
\cline { 2 - 3 } & $\mathbf{M C F}-7$ & $\mathbf{C a S k i}$ \\
\hline $\mathbf{2}$ & $-^{\mathrm{a}}$ & $22.81 \pm 1.33$ \\
$\mathbf{3}$ & $17.30 \pm 2.11$ & $24.44 \pm 0.22$ \\
$\mathbf{4}$ & $20.10 \pm 1.52$ & $25.59 \pm 1.70$ \\
$\mathbf{5}$ & $14.47 \pm 0.51$ & $21.95 \pm 2.56$ \\
Doxorubicin & $0.06 \pm 0.01$ & $0.20 \pm 0.02$ \\
\hline & ${ }^{\mathrm{a}} \mathrm{IC}_{50}>10 \mu \mathrm{g} / \mathrm{mL}$.
\end{tabular}

\section{Experimental Section}

\subsection{General Experimental Procedures}

Optical rotations were measured on a Perkin-Elmer 341 polarimeter. UV spectra were recorded on a Shimadzu UV-160 UV-visible spectrometer (Shimadzu, Kyoto, Japan). NMR spectra were acquired on a Varian Mercury-400 Plus NMR spectrometer (Varian, CA, USA) with TMS as internal standard. HRESIMS was carried out on a micrOTOF-Q II ESI mass spectrometer (Bruker, Bremen, Germany). Single-crystal X-ray diffraction analysis was performed on a Bruker APEX II diffractometer (Bruker, Bremen, Germany).

\subsection{Fungal Material and Fermentation}

The fungus AS21B used in the present study was isolated from leaves of Azima sarmentosa, collected from the mangrove forest in Samutsakhon province, Thailand in July 2008. The fungus was identified as a Rhytidhysteron sp. based on the ITS sequences, and was deposited at Department of Chemistry, Faculty of Science, Chulalongkorn Universtiy. The strain AS21B was grown on potato dextrose agar (PDA) plate at room temperature for 7 days. Five pieces $\left(5 \times 5 \mathrm{~mm}^{2}\right)$ of mycelial agar plugs were inoculated into $1 \mathrm{~L}$ Erlenmeyer flasks $(\times 50)$ containing $200 \mathrm{~mL}$ of malt extract broth (MEB). The cultivation was kept at room temperature for 21 days under static conditions.

\subsection{Extraction and Isolation}

The mycelia were separated off from the broth by filtration. The filtrate was extracted with an equal amount of EtOAc for 3 times. The EtOAc solution was evaporated under reduced pressure to afford a crude extract $(7.0 \mathrm{~g})$. The extract was subjected to a Sephadex LH20 column and eluted with $\mathrm{MeOH}$ to give six fractions (F1-F6). Subsequently, fraction 5 was fractionated by silica gel $\left(\mathrm{SiO}_{2}\right)$ column chromatography eluted by a gradient of $\mathrm{MeOH} / \mathrm{CH}_{2} \mathrm{Cl}_{2}$ from 1:99 to 1:9 to yield nine subfractions. The fraction F5.3 was purified by $\mathrm{SiO}_{2}$ column chromatography (a gradient of EtOAc/hexane from 2:8 to $1: 1)$ to give $3(20.1 \mathrm{mg})$ and $4(47.8 \mathrm{mg})$. Subfraction F5.4 was rechromatographed on $\mathrm{SiO}_{2}$ with a 1:1 mixture of EtOAc/hexane to obtain $2(13.5 \mathrm{mg})$. Fraction F5.6 was applied to $\mathrm{SiO}_{2}$ column chromatography eluted with $\mathrm{MeOH} / \mathrm{CH}_{2} \mathrm{Cl}_{2}(1: 19)$ to afford $5(15.2 \mathrm{mg})$. Fraction F5.7 was divided into four fractions by column chromatography on Sephadex LH20 (MeOH), then F5.7.4 was further purified by $\mathrm{SiO}_{2}$ column chromatography with $\mathrm{MeOH} / \mathrm{CH}_{2} \mathrm{Cl}_{2}$ (1:9) to give 1 (83 mg). Fraction F6 was 
subjected to a Sephadex LH20 column eluted with $\mathrm{MeOH}$ to yield five subfractions. F6.4 was recrystallized with $\mathrm{MeOH}$ to provide $7(32.6 \mathrm{mg})$. Subfraction F6.5 was rechromatographed over a $\mathrm{SiO}_{2}$ column to give three fractions. The fraction $\mathrm{F} 6.5 .3$ was further purified by $\mathrm{SiO}_{2}$ column chromatography eluted with EtOAc/hexane (1:3) to yield $6(12.8 \mathrm{mg})$ and 8 (8.2 $\mathrm{mg})$.

Rhytidone A (1): light brown powder; $[\alpha]^{25}{ }_{\mathrm{D}}+21(c 0.10, \mathrm{MeOH}) ;{ }^{1} \mathrm{H}$ and ${ }^{13} \mathrm{C}$ NMR, see Table 1; HRESIMS $m / z 381.1319[\mathrm{M}+\mathrm{Na}]^{+}$(calcd for $\mathrm{C}_{20} \mathrm{H}_{22} \mathrm{O}_{6} \mathrm{Na}, 381.1314$ ).

Rhytidone B (2): colorless crystals; $[\alpha]^{25}+15$ (c $\left.0.10, \mathrm{MeOH}\right) ;{ }^{1} \mathrm{H}$ and ${ }^{13} \mathrm{C}$ NMR, see Table 1 ; HRESIMS $m / z 379.1153[\mathrm{M}+\mathrm{Na}]^{+}$(calcd for $\mathrm{C}_{20} \mathrm{H}_{20} \mathrm{O}_{6} \mathrm{Na}, 379.1158$ ).

Rhytidone C (3): colorless crystals; $[\alpha]^{25}{ }_{\mathrm{D}}+18$ (c $\left.0.10, \mathrm{MeOH}\right) ;{ }^{1} \mathrm{H}$ and ${ }^{13} \mathrm{C}$ NMR, see Table 1; HRESIMS $m / z$ 393.1315 [M+ Na] $]^{+}$(calcd for $\mathrm{C}_{21} \mathrm{H}_{22} \mathrm{O}_{6} \mathrm{Na}, 393.1314$ ).

Preparation of 5-O-Acetyl-rhytidone A (1a). A mixture of compound 1 (10 mg), acetic anhydride $(0.1 \mathrm{~mL})$ and a catalytic amount of DMAP in $\mathrm{CH}_{2} \mathrm{Cl}_{2}(2 \mathrm{~mL})$ was stirred at room temperature for $30 \mathrm{~min}$. Then, water $(5 \mathrm{~mL})$ was added to the reaction mixture, and extracted with EtOAc $(3 \times 5 \mathrm{~mL})$. The combined organic layer was washed with brine, dried over $\mathrm{MgSO}_{4}$, and evaporated after filtration. The residue was purified by $\mathrm{SiO}_{2}$ column chromatography $\left(\mathrm{MeOH} / \mathrm{CH}_{2} \mathrm{Cl}_{2}, 2: 98\right)$ to yield $\mathbf{1 a}(7.2 \mathrm{mg}$, $64 \%)$ as colorless crystals: ${ }^{1} \mathrm{H} \mathrm{NMR}\left(\mathrm{CDCl}_{3}, 400 \mathrm{MHz}\right) \delta 7.51\left(1 \mathrm{H}, \mathrm{d}, J=8.4 \mathrm{~Hz}, \mathrm{H}-5^{\prime}\right), 7.47(1 \mathrm{H}, \mathrm{d}$, $\left.J=8.4 \mathrm{~Hz}, \mathrm{H}-4^{\prime}\right), 7.44\left(1 \mathrm{H}, \mathrm{t}, J=7.2 \mathrm{~Hz}, \mathrm{H}-6^{\prime}\right), 7.39\left(1 \mathrm{H}, \mathrm{t}, J=7.2 \mathrm{~Hz}, \mathrm{H}-3^{\prime}\right), 6.93(1 \mathrm{H}, \mathrm{d}, J=8.4 \mathrm{~Hz}$, H-2'), $6.906 .90\left(1 \mathrm{H}, \mathrm{d}, J=8.4 \mathrm{~Hz}, \mathrm{H}-7^{\prime}\right), 5.34(1 \mathrm{H}, \mathrm{ddd}, J=5.2,10.0,16.8 \mathrm{~Hz}, \mathrm{H}-5), 4.56(1 \mathrm{H}$, br s, $\mathrm{H}-8), 4.13$ (1H, br m, H-7), $3.88(1 \mathrm{H}$, br s, 4-OH), $3.70(1 \mathrm{H}, \mathrm{s}, 8-\mathrm{OH}), 3.58(1 \mathrm{H}$, br s, H-4), $2.76(1 \mathrm{H}$, dd, $J=1.6,12.8 \mathrm{~Hz}, \mathrm{H}-8 \mathrm{a}), 2.43$ (1H, ddd, $J=2.0,12.8,16.8 \mathrm{~Hz}, \mathrm{H}-4 \mathrm{a}), 2.27$ (1H, ddd, $J=2.4,12.8$, $14.4 \mathrm{~Hz}, \mathrm{H}-6 \mathrm{a}), 2.15$ (3H, s, 5-OAc), 2.00 (1H, m, H-6b), 1.97 (2H, m, H-2), 1.73 (2H, m, H-2); ${ }^{13} \mathrm{C} \mathrm{NMR}\left(\mathrm{CDCl}_{3}, 100 \mathrm{MHz}\right) \delta 172.7\left(5-\mathrm{OCOCH}_{3}\right), 147.5\left(\mathrm{C}-8^{\prime}\right), 146.0\left(\mathrm{C}-1^{\prime}\right), 134.2\left(\mathrm{C}-4 \mathrm{a}^{\prime}\right), 127.7$ (C-6'), 127.0 (C-3'), 121.3 (C-5'), 120.3 (C-4'), 113.9 (C-8a'), 109.8 (C-2'), 109.5 (C-7'), 104.7 (C-1), 69.4 (C-5), 69.2 (C-7), 67.6 (C-8), 62.6 (C-4), 41.3 (C-4a), 39.0 (C-8a), 32.3 (C-6), 27.2 (C-3), $25.6(\mathrm{C}-2), 21.2\left(5-\mathrm{OCOCH}_{3}\right)$.

X-ray Crystallographic Analysis of compounds 1a, 2 and 3. All single crystal X-ray diffraction data were collected at $296 \mathrm{~K}$ on a Bruker APEX II diffractometer with Mo K $\alpha$ radiation $(\lambda=0.71073 \AA)$. The structures were solved by direct methods using SHELXS-97 and refined full-matrix least squares on all $F^{2}$ data using SHELXL97 to final $R$ values [18,19]. All hydrogen atoms were added at calculated positions and refined using a rigid model. Crystallographic data for 1a, $\mathbf{2}$ and $\mathbf{3}$ have been deposited with the Cambridge Crystallographic Data Centre (Cambridge, UK) [20].

Crystal data of 1a: colorless crystal; $\mathrm{C}_{22} \mathrm{H}_{24} \mathrm{O}_{7}, M_{r}=400.41$, monoclinic, $a=12.220(2) \AA$, $b=5.7498(9) \AA, c=13.925(3) \AA$, space group $P 2_{1}, Z=2$ and $V=956.2(3) \AA^{3}, \mu($ Mo Ka $)=0.10 \mathrm{~mm}^{-1}$, and $F(000)=424$. Crystal dimensions: $0.42 \times 0.27 \times 0.25 \mathrm{~mm}$. Independent reflections: 1492 $\left(R_{\text {int }}=0.063\right)$. The final $R_{1}$ values were $0.039, \mathrm{w} R_{2}=0.113(I>2 \sigma(I))$. CCDC number: 977804 .

Crystal data of 2: colorless crystal; $\mathrm{C}_{20} \mathrm{H}_{20} \mathrm{O}_{6}, M_{r}=356.36$, orthorhombic, $a=7.6970(6) \AA$, $b=8.4012(8) \AA, c=25.845(2) \AA$, space group $P 2{ }_{1} 2{ }_{1} 2_{1}, Z=4$ and $V=1617.3(2) \AA^{3}$, $\mu(\mathrm{Mo} \mathrm{K} \alpha)=0.11 \mathrm{~mm}^{-1}$, and $F(000)=752$. Crystal dimensions: $0.40 \times 0.22 \times 0.20 \mathrm{~mm}$. Independent 
reflections: $3200\left(R_{\text {int }}=0.025\right)$. The final $R_{1}$ values were $0.037, \mathrm{w} R_{2}=0.110(I>2 \sigma(I))$. CCDC number: 977805.

Crystal data of 3: colorless crystal; $\mathrm{C}_{21} \mathrm{H}_{22} \mathrm{O}_{6}, M_{r}=370.39$, monoclinic, $a=23.9484(6) \AA$, $b=5.6338(1) \AA, c=15.5486(4) \AA$, space group $P 2{ }_{1} 2_{1} 2_{1}, Z=4$ and $V=1783.37(7) \AA^{3}$, $\mu(\mathrm{Mo} \mathrm{K} \alpha)=0.10 \mathrm{~mm}^{-1}$, and $F(000)=784$. Crystal dimensions: $0.36 \times 0.34 \times 0.24 \mathrm{~mm}$. Independent reflections: $3133\left(R_{\text {int }}=0.016\right)$. The final $R_{1}$ values were $0.037, \mathrm{w}_{2}=0.119(I>2 \sigma(I))$. CCDC number: 898711 .

\subsection{Cytotoxicity Assay}

Cytotoxicity of all compounds was assayed with a modification of the MTT (3-[4,5-dimethylthiazol-2-yl-2,5-diphenyltetrazolium] bromide) colorimetric method. Cytotoxicity assays were performed according to previously described procedures [16]. The following human cancer cell lines were used in the assay: human breast cancer (MCF-7) and cervical carcinoma (CaSki) cell lines. Doxorubicin was used as the reference compound.

\section{Conclusions}

The chemical investigation of the EtOAc extract of the endophytic fungus Rhytidhysteron sp. has led to the isolation and characterization of three spirobisnaphthalenes, along with five known derivatives. All isolated compounds (1-8) were evaluated for their cytotoxicity against breast and cervical cancer cells. Compounds 3-5 exhibited cytotoxicity against both cell lines, whereas compound 2 was active only for cervical carcinoma cells.

\section{Acknowledgments}

This work was supported by the Thailand Research Fund (RSA5580023), and the Ratchadaphiseksomphot Endowment Fund of Chulalongkorn University (RES560530208-AS). The authors also gratefully thank Miss Sujitra Hantanong, Program in Biotechnology, Faculty of Science, Chulalongkorn University, for fungal cultivation.

\section{Conflicts of Interest}

The authors declare no conflict of interest.

\section{References}

1. Jiao, P.; Swenson, D.C.; Gloer, J.B.; Campbell, J.; Shearer, C.A. Decaspirones A-E, bioactive spirodioxynaphthalenes from the freshwater aquatic fungus Decaisnella thyridioides. J. Nat. Prod. 2006, 69, 1667-1671.

2. Hu, H.; Guo, H.; Li, E.; Zhou, Y.; Che, Y. Decaspirones F-I, bioactive secondary metabolites from the saprophytic fungus Helicoma viridis. J. Nat. Prod. 2006, 69, 1672-1675. 
3. Macías-Rubalcava, M.L.; Hernández-Bautista, B.E.; Jiménez-Estrada, M.; González, M.C.; Glenn, A.E.; Hanlin, R.T.; Hernández-Ortega, S.; Saucedo-García, A.; Muria-González, J.M.; Anaya, A.L. Naphthaquinone spiroketal with allelochemical activity from the newly discovered endophytic fungus Edenia gomezpompae. Phytochemistry 2008, 69, 1185-1196.

4. Matínez-Luis, S.; Della-Togna, G.; Coley, P.D.; Kursar, T.A.; Gerwick, W.H.; Cubilla-Rios, L. Antileishmanial constituents of the Panamanian endophytic fungus Edenia sp. J. Nat. Prod. 2008, 71, 2011-2014.

5. Van der Sar, S.A.; Blunt, J.W.; Munro, M.H.G. Spiro-Mamakone A, a unique relative of the spironaphthalene class of compounds. Org. Lett. 2006, 8, 2059-2061.

6. Chen, X.; Shi, Q.; Lin, G.; Guo, S.; Yang, J. Spirobisnaphthalene analogues from the endophytic fungus Preussia sp. J. Nat. Prod. 2009, 72, 1712-1715.

7. Cai, Y.-S.; Guo, Y.-W.; Krohn, K. Structure, bioactivities, biosynthetic relationships and chemical synthesis of the spirodioxynaphthalenes. Nat. Prod. Rep. 2010, 27, 1840-1870.

8. Zhang, H.W.; Song, Y.C.; Tan, R.X. Biology and chemistry of endophytes. Nat. Prod. Res. 2006, $23,753-771$.

9. Gunatilaka, A.A.L. Natural products from plant-associated microorganisms: Distribution, structural diversity, bioactivity and implications of their occurrence. J. Nat. Prod. 2006, 69, 509-526.

10. Strobel, G.; Diasy, B.; Castillo, U.; Harper, J. Natural products form endophytic microorganisms. J. Nat. Prod. 2004, 67, 257-268.

11. Strobel, G.A. Endophytes as sources of bioactive products. Microbes Infect. 2003, 5, 535-544.

12. Debbab, A.; Aly, A.H.; Proksch, P. Mangrove derived fungal endophytes-A chemical and biological perception. Fungal Divers. 2013, 61, 1-27.

13. Sakemi, S.; Inagaki, T.; Kaneda, K.; Hirai, H.; Iwata, E.; Sakakibara, T.; Yamauchi, Y.; Norcia, M.; Wondrack, L.M.; Sutcliffe, J.A.; Kojima, N. CJ-12,371 and CJ-12,372, two novel DNA gyrase inhibitors, fermentation, isolation, structural elucidation and biological activities. J. Antibiot. 1995, 48, 134-142.

14. Wipf, P.; Lynch, S.M.; Birmingham, A.; Tamayo, G.; Jiménez, A.; Campos, N.; Powis, G. Natural product based inhibitors of the thioredoxin-thioredoxin reductase system. Org. Biomol. Chem. 2004, 2, 1651-1658.

15. Ragot, J.P.; Steeneck, C.; Alcaraz, M.-L.; Taylor, R.J.K. The synthesis of 1,8-dihydroxynaphthalene-derived natural products: Palmarymycin $\mathrm{CP}_{1}$, Palmarymycin $\mathrm{CP}_{2}$, Palmarymycin $\mathrm{CP}_{11}$, CJ-12,371, deoxypreussomerin A and novel analogues. J. Chem. Soc. Perkin Trans. 1 1999, 1073-1082; doi:10.1039/A901076I.

16. Ohishi, H.; Chiba, N.; Mikawa, T.; Sakaki, T.; Miyaji, S.; Sezaki, M. Novel Antibiotic MK3018 substance_-Useful as antimicrobial agent. Jpn. Pat. 01294686, 28 November 1989.

17. Chokpaiboon, S.; Sommit, D.; Teerawatananond, T.; Muangsin, N.; Bunyapaiboonsri, T.; Pudhom, K. Cytotoxic nor-chamigrane and chamigrane endoperoxides from a basidiomycetous fungus. J. Nat. Prod. 2010, 73, 1005-1007.

18. Sheldrick, G.M. SHELXS-97, Program for Crystal Structure Solution; University of Göttingen: Göttingen, Germany, 1997. 
19. Sheldrick, G.M. SHELXS-97, Program for Crystal Structure Refinement; University of Göttingen: Göttingen, Germany, 1997.

20. Cambridge Crystallographic Data Centre. Available online: http://www.ccdc.cam.ac.uk/ data_request/cif (accessed on 24 February 2014).

(C) 2014 by the authors; licensee MDPI, Basel, Switzerland. This article is an open access article distributed under the terms and conditions of the Creative Commons Attribution license (http://creativecommons.org/licenses/by/3.0/). 